\title{
Les instants des rencontres culturelles dans Montréal et leurs impacts social et national
}

\section{Moments of cultural encounters in Montreal and their social and national impacts}

Patrick Imbert ${ }^{1}$

Submetido em 2 e aprovado em 13 de novembro de 2017. Résumé: L'homogénéité culturelle évoquée dans les romans de la terre se dissout de plus en plus dans la littérature québécoise liée à la ville, surtout depuis les années 1970, lorsque s'explorent la rencontre et les altérités. Ainsi, se repensent l'identité et la manière de la légitimer par la durée longue, à travers la valorisation de l'impact de l'instant de la rencontre.

Mots clés: Ville. Rencontre. Altérité. Instant. Montréal.

Abstract: Cultural homogeneity evoked by the traditional Québec novel loses its importance in the 1970s, as writers start exploring the encounter between different cultural groups in an urban context. Thus, the conception of identity defined by the long term starts changing as well through the assessment of the instant of the encounter.

Keywords: Urbanity. Encounter. Alterity. Instant. Montréal.

\section{Introduction}

Qu'est ce qu'une ville? C'est un espace restreint et réseauté qui impose des parcours répétés faits d'habitudes quotidiennes menant à être socio-économiquement productif. Elle est aussi le lieu des croisements temporaires entre des hiérarchies sociales qui circulent selon des itinéraires séparés mais qui se manifestent dans des nœuds de production, de capitalisation et de consommation.

Au Canada français, Gabrielle Roy est la première à explorer la ville de façon approfondie dans Bonheur d'occasion. C'est le premier roman qui casse l'homogénéité collective telle qu'elle s'est manifestée dans le roman de la terre montrant le village tricoté, serré autour du clocher et perpétuant le rêve de Philippe Aubert de Gaspé père affiché dans Les anciens Canadiens, celui d'un Canadien français cousu d'unanimisme optimiste. Celui- 
ci était menacé seulement par l'étranger, anglais ou immigrant, comme on le voit dans $L a$ terre paternelle de Patrice Lacombe en 1845 ou dans Charles Guérin de Pierre-JosephOlivier Chauveau en 1852. N'oublions pas non plus la célèbre phrase d'Edmond de Nevers affirmant que la question sociale est la question nationale: «La question sociale n'existe pas au Canada et ne saurait exister dans les conditions actuelles de notre vie économique» (p. 185). Elle est reprise en 1966 par Pierre de Grandpré dans Dix ans de vie littéraire au Canada français: «En ce pays, la question sociale, c'est la question nationale, il n'y en a pas d'autre» (p. 42). Ainsi, l'unanimisme collectif prime à la fois sur l'affirmation individuelle et sur la division en classes sociales ce qui est corroboré par une affirmation telle que la suivante tirée encore d'Edmond de Nevers dans L'avenir du peuple canadienfrançais: « Notre nationalité résisterait à l'oppression, elle succombera par la tolérance, si nous ne nous hâtons pas d'ouvrir des champs nouveaux à l'activité des esprits, à l'ardeur des tempéraments » (p.14).

Croire que la question sociale, c'est la question nationale est répandu dans les romans qui perpétuent, jusqu'à la Deuxième Guerre mondiale, l'imaginaire du village alors même que la majorité des Québécois vivent en ville. D'ailleurs jusqu'à récemment, articuler question sociale et question nationale reste difficile comme le souligne par exemple le texte intitulé «De la question sociale à la question nationale: la revue Cité Libre (1950-1963)» d'E.-Martin Meunier et Jean-Philippe Warren.

Ainsi, une écrivaine de l'Ouest - Gabrielle Roy - connaissant les traditions de gauche illustrées par un politicien du Manitoba comme Tommy Douglas qui a mis en place le premier régime de santé universel et gratuit au Canada, parle pour la première fois de manière élaborée dans un roman, de Montréal comme une ville clivée entre Saint-Henri et Westmount, le quartier francophone et le quartier anglophone. Le clivage, toutefois reste en bonne partie ethnique et non pas de classe. Pourtant, Outremont bourgeois et francophone ne communique pas plus avec Saint-Henri. Ainsi, le clivage social est aussi un clivage national comme le souligne Pierre de Grandpré. Il va servir à penser la ville en fonction des grands mouvements de décolonisation appuyés sur le marxisme. Le portrait du colonisé d'Albert Memmi sera publié à Montréal en 1972, les discours inspirés des mouvements de libération nationale vont être populaires dans les années 1960 et 1970. 
Ils vont traduire l'aspiration à la lutte des classes en tant qu'elle se fabrique dans des contextes historico-nationaux restreints, la révolution se faisant par fragments et non globalement comme le rêvait Trotsky assassiné sur ordre de Staline à Mexico le 20 août 1940. Cet amalgame réel certes mais non exclusif entre classe et ethnicité se manifeste bien dans divers textes publiés dans ces mêmes années. On pense à Pierre Vallières qui, dans son livre intitulé Nègres blancs d'Amérique, pose les Francophones comme minorité opprimée mais pas opprimante, les Autochtones étant constamment oubliés et les Noirs invisibilisés. Certes, on ne niera pas, comme l'ont montré bien des auteurs, de Gabrielle Roy dans Bonheur d'occasion à Paul Chamberland dans L'afficheur hurle, que les Canadiens français minoritaires étaient relégués dans les marges de l'État canadien. Toutefois, il faut retenir ce que Vallières dit des Noirs au Québec: «Au Québec, les Canadiens français ne connaissent pas ce racisme irrationnel qui a causé tant de tort aux travailleurs blancs et aux travailleurs noirs des États-Unis. Ils n'ont aucun mérite à cela, puisqu'il n'y a pas, au Québec, de 'problème noir'» (p. 26). On voit alors le fonctionnement d'un dualisme omniprésent qui amalgame classe et ethnicité. ${ }^{2}$ Les barbares sont les gens des États-Unis à cause du racisme féroce qu'on y rencontre, ce qui a mené au mouvement des droits civiques. Pour Pierre Vallières, les barbares sont les bourgeois capitalistes anglophones qu'il faut éliminer. Les civilisés sont les gens du peuple, les Francophones exploités. Ce dualisme empêche de voir les Noirs au Québec et leur situation vécue dans l'exclusion, alors qu'il y a de nombreux Noirs nés au Canada depuis l'arrivée des esclaves qui travaillaient pour des Canadiens français comme 1'a rappelé Marcel Trudel en 1963 dans L'esclavage au Canada français. De plus, beaucoup de Noirs sont arrivés au Canada au $19^{\text {ème }}$ siècle fuyant les États-Unis et se sont installés dans le sud de l'Ontario ou à Halifax comme le mentionne George Elliott Clarke dans Execution poems en 2001. Il n’y a pas de problème noir car ils sont peu nombreux et n'ont pas voix au chapitre. C'est pourtant le contraire que souligne Lorena Gale, une montréalaise noire et anglophone dans «Je me souviens»: 'I remember when they wouldn't serve us in the restaurants on St.Catherine's St. Or let us in the movie theatres». ${ }^{3}$ Ce n'est que dans les années 1980 que Dany Laferrière posera humoristiquement le problème en 1985 dans Comment faire l'amour avec un Nègre sans se fatiguer et ce, dans un contexte nouveau, celui d'une prise 
de conscience des différences, du racisme, de l'immigration, du multiculturalisme, de l'interculturalisme et des stéréotypies négatives.

\section{Les clivages urbains}

Ainsi, la ville repose sur des clivages ethnicisés et nationaux mâtinés de colonialisme, ce que souligne aussi Jacques Ferron dans «Rencontre au Kentucky», une nouvelle tirée des Contes anglais. Dans ce conte, il s'agit d'un «Amerloque» qui se bat en Europe pour que celle-ci retrouve la démocratie face aux horreurs nazies, fascistes et staliniennes, ce que Ferron ne précise pas en restant dans la généralité de la guerre qui sévit. Cet Amerloque rencontre une Belge francophone, la séduit et l'épouse. Observons la description de la rencontre: «L'Amerloque, lui, avait joué au sauvage toute son enfance, puis était allé au cinéma y apprendre son monde, un monde à deux-dimensions où faute de la troisième tout était illusoire» (1985, p. 165). Parler d'un Amerloque est déjà catégoriser quelqu'un comme inadéquat, ce qui se confirme avec la liste des activités qui lui confèrent l'attribut de sauvage reprenant le célèbre paradigme dualiste barbarie/civilisation tiré des savants européens du $19^{\text {ème }}$ siècle et diffusé par Sarmiento dans Facundo en 1845. Il est sauvage car il ne connait ni le monde, ni la littérature, mais Hollywood. Cette présentation de Ferron est surprenante car un soldat au front ne doit certainement pas avoir vécu la guerre comme dans un film, vu l'horreur des blessures, des morts et des bombardements! Le plus intéressant est à venir. Il ne veut pas apprendre le français et sa femme prise, quant à elle, dans les luttes linguistiques français/flamand ne parvient pas à apprendre l'anglais. Ils choisissent de s'établir au Canada, dans la banlieue de Montréal car c'est un endroit qui s'accommode «de nos déficiences linguistiques» (p. 166). Ainsi, parce que le lieu est accommodant, c'est-à-dire dans ce contexte de pensée, non-homogène et faible identitairement, il tolère, par son vide, toutes les inadéquations. Les parents sont d'ailleurs qualifiés de «Toqués» (p. 166). Quant aux enfants, on affirme «Ce sont eux qui vous apprendront l'anglais, ma chère!» (p. 166). Chez Ferron, les enfants de parents non québécois vont recommencer à neuf en s'appuyant sur la langue dominante, celle du père anglophone. Il reste toutefois un autre point chez Ferron: «Que sommes-nous sinon une marche de l'Europe en Amérique. L'Europe a été perdante. Il était assez naturel qu'un soldat américain vînt achever chez nous 
sa victoire ${ }^{4}$. Chez Ferron qui parle après la Deuxième Guerre mondiale, les barbares étatsuniens ont gagné et menacent le Canada-français, européen de tradition et sa civilisation. La ville est un lieu d'assimilation aux divisions ethniques marquées, soumise aux menaces de l'impérialisme qui vise l'homogénéité de la culture québécoise jamais présentée dans ses clivages économiques à l'intérieur même de la collectivité francophone. Voilà une vision somme toute peu nouvelle par rapport à la tradition littéraire canadienne française.

\section{Le consensus socio-national et les origines littéraires}

Le consensus social cherché avec avidité s'affirme dès les origines de la littérature au Canada français. On sait que le premier roman publié, en 1837, trois mois avant L'influence d'un livre de Philippe Aubert de Gaspé fils est Cambray et ses complices de François-Réal Angers. Il s'agit d'un commerçant canadien-français qui, entre autre, pille les églises. Le livre a disparu de la mémoire littéraire et a été remplacé comme livre des origines dans les anthologies par La terre paternelle de Patrice Lacombe (1845) ou même par Les anciens Canadiens de Philippe Aubert de Gaspé, père (1867). Ce n'est que le critique francophone de l'Ontario Séraphin Marion qui rétablit l'origine dans Les lettres canadiennes d'autrefois. Il affirme que le premier roman est L'influence d'un livre de Philippe Aubert de Gaspé, fils. Cet ouvrage a toutefois été censuré car il ne montre pas une vision consensuelle et religieuse de la société québécoise. Mais il reste le cas de Cambray et ses complices. Même de nos jours, certains critiques ne le reconnaissent pas comme premier roman car il est composé en partie de longues citations du procès réel fait à ce malfaiteur. Toutefois, on voit mal pourquoi ce roman témoignage et orienté sur la loi devrait être exclu puisqu'à l'époque un ouvrage comme Les anciens Canadiens est composé de quantité d'anecdotes,

de chansons et de références diverses. De plus, à l'époque encore, la littérature était un ensemble de textes écrits bien moins codifiés que de nos jours.

N'oublions pas non plus qu'en 1835, Le père Goriot de Balzac a été publié en feuilleton de façon très censurée dans un journal intitulé L'ami du peuple, de l'ordre et des lois $^{5}$. Comme le dit Dany Laferrière, quand je suis lu par des Japonais, je suis un écrivain japonais. Quand Le père Goriot est lu et encore plus censuré, c'est-à-dire réécrit par des Canadiens français, Le père Goriot devient un texte Canadien français. Dans ce cas, 
d'ailleurs, la censure de la fin où Rastignac dit, face à Paris, maintenant à nous deux, est symptomatique d'une lecture d'ici. En effet, ce qui est refusé est l'attitude d'un individu qui entre en lutte contre la société, contre la ville. Pour l'institution littéraire et politique, l'affirmation individuelle qui plus est, contre le groupe, est un très mauvais exemple. Ainsi, dans le feuilleton à Montréal, Rastignac suit, résigné, le corbillard où repose le Père Goriot. Comme pour Blanche d'Haberville qui, dans Les anciens Canadiens, n'est pas un individu mais représente le groupe canadien français dans son ensemble et n'épousera jamais Archibald malgré son attirance pour lui, le Rastignac montréalais représente le groupe et pas un individu qui s'affirme. La ville est donc présentée par l'activité censurante comme un village où tous se soumettent et collaborent pour maintenir le consensus. Il faut croire à une égalité devant Dieu et à des différences mineures ici-bas. Pas question d'évoquer des fractures sociales à l'intérieur du groupe canadien français.

\section{Les fractures sociales et nationales}

Toutefois, récemment, c’est-à-dire au moment où les écrivains non nés au Québec pénètrent le centre littéraire et publient chez les éditeurs importants à Montréal, une autre dynamique se met en place.

Parler de la ville, dans ces textes, mène à considérer que le clivage social est omniprésent. Toutefois, chez Dany Laferrière il n'est pas encore présenté entre Blancs francophones et on ne montre pas encore que la société présentée comme unanime et homogène, est en fait un vivier de concurrence interne comme le soulignent à la fois les marxistes et un anthropologue comme René Girard (1978). Dans Comment faire l'amour avec un Nègre sans se fatiguer, Dany Laferrière montre bien les difficiles relations entre groupes sociaux dans la ville de Montréal. Toutefois, il se garde bien de toucher au collectif francophone. Les étudiantes bourrées d'hormones et de stéréotypes au sujet des Noirs sont des Anglophones de McGill et elles vivent dans des quartiers riches. Certes, ces jeunes filles échappent aux parcours traditionnels et vont explorer le taudis d'un immigrant haïtien. Certes, elles explorent les différences sociales et similairement pour l'écrivain haïtien. Mais la rencontre profonde, dormir ensemble, manifester sa faiblesse, se mettre à nu et se révéler

vulnérable n'est pas au rendez-vous. De plus, la société francophone n'est présente que 
comme lectrice potentielle de rapports stéréotypés entre anglo-montréalaises et immigrants Noirs. Rien pour poser la question d'un clivage quelconque au sein de la société francomontréalaise dans cet ouvrage qui devient un best-seller.

Il est intéressant de saisir que le clivage social organisé seulement selon des lignes ethniques se brise quelque peu dans le roman d'Elena Botchorichvili intitulé Seulement attendre et regarder. Dans ce cas, il s'agit d'immigrés des anciens pays colonisés par l'Union soviétique. Ils vivent à Westmount dans le quartier riche et anglophone chez un québécois francophone qui les héberge. Le clivage riche anglophone/pauvre francophone ne joue plus. Les classes sociales ne sont plus divisées selon des lignes uniquement ethnolinguistiques. De même, les immigrés rejettent tout héritage soviétique dont évidemment la conception d'une division forte entre classes sociales et son corolaire la lutte des classes. Ils sont pris entre nostalgie et incompréhension mais certains finissent par apprivoiser le fonctionnement socioéconomique du Québec. Ainsi, c'est au moment où l'idée de lutte des classes et de classes rigidement séparées disparaît que se transforme et se complexifie aussi le clivage économique entre Anglophones et Francophones. Tous deux peuvent être riches ou pauvres. Autrement dit, quand la question sociale se transforme sous l'influence d'une prise de parole d'écrivains liés à d'autres perspectives, la question nationale se complexifie nettement. Ainsi, la question sociale est liée à la question nationale certes, mais elle n'est pas uniquement la question nationale.

C'est d'ailleurs ce qui est aussi exploré dans La logeuse d'Éric Dupont. On y voit une Gaspésienne fuyant son village Notre-Dame-du-Cachalot opprimé par une homogénéisation forcée marxo-linguistico-ethnique qui transforme le village en prison. Elle le quitte pour Montréal où elle a pour amies des immigrantes qui travaillent comme amuseuses et prostituées. Plusieurs sont originaires des pays de l'Est et le clou du spectacle de leur groupe nommé Les Arrière-Petites-Filles de Lénine est de chanter ironiquement l'Internationale. Dans ce cas, les clivages sociaux sont modifiés par un capitalisme débridé qui n'est plus contextualisé avec la lutte des classes. Voilà qui entraine des rencontres profondes entre les immigrantes et la migrante de Gaspésie qui se rendent compte qu'au delà de leur différences, elles vivent des conditions de travail difficiles et similaires. Autrement dit, la question sociale ne se pose plus en terme de lutte des classes vu l'échec du marxisme et la 
question nationale, parodiée sous les activités bureaucratico-linguistiques de Jeanne Joyal, la logeuse nationaliste ne nie plus la possibilité de créer des liens citoyens qui dépassent les origines ethniques ou le statut d'immigrant.

Dès lors, c'est-à-dire chez Elena Botchorichvili comme chez Éric Dupont, la question sociale n'est pas la question nationale. Ou plutôt, elle peut l'être dans l'optique des immigrantes illégales qui désirent rester ici et devenir citoyennes et qui sont retournées dans leur pays où il arrive qu'elles se suicident comme Shu Mysty. C'est aussi ce qu'évoque Alexandre, le personnage principal chez Yvon Rivard lorsqu'il évoque Montréal: «Le Nouveau Monde, c'est aussi cela, bien sûr des corps, des vitrines, des marchandises, un monde de nouveaux riches qui ne se souviennent plus de leur pauvreté, un monde de pauvres qui ne rêvent que d'être riches» (p. 291). Ici aussi, la question sociale quoique abordée rapidement ne se greffe pas immédiatement à la question nationale. Il y a des riches et des pauvres dans le Nouveau Monde et ils rêvent à un monde meilleur comme Vieux, l'immigrant de Comment faire l'amour avec un Nègre sans se fatiguer qui veut l'Amérique toute entière et qui refuse le repli ou le ghetto urbain. Dans le mélange, dans les rencontres au hasard, des solidarités se tissent dans le complexe d'instants à partager dans le présent audelà des passés qui divisent et des idéologies, qu'elles soient nationalistes ou communistes. En effet, celles-ci n'ont pas su créer les conditions de solidarités dépassant les exclusions flagrantes, celles des femmes, des immigrantes, des dissidents.

\section{L'instant plutôt que la durée longue}

En ce sens, ces textes récents sont dissidents par rapport à la volonté dans le roman canadien français traditionnel d'imposer un consensus ethnolinguistique qui nie les fractures économiques et sociales complexes. Le but était de parvenir à occuper un espace menacé et d'imposer une durée longue par la référence aux ancêtres qui occupent le territoire depuis des siècles. De nos jours, toutefois, ce qui compte de plus en plus est l'instant ainsi qu'on le voit dans Le siècle de Jeanne d'Yvon Rivard. Un siècle qui valorise non la durée longue mais l'instant de la rencontre.

Dans Le siècle de Jeanne, Yvon Rivard procède à une longue réflexion sur les Amériques et affirme qu'on peut y être oisif et sauvage et que ces qualificatifs fondent la 
créativité des gens des Amériques. Ces deux attributs qui définissaient quelqu'un comme barbare dans l'optique de Sarmiento ou même dans le conte de Ferron, sont maintenant redéfinis. Être oisif pour Yvon Rivard, c'est se mettre en état de disponibilité pour chaque être, pour ce qui est différent et pour chaque paysage et situation dont on peut mesurer la beauté car il y a là, comme il le rappelle, de la vie qui pourrait ne pas/ne plus exister. Être sauvage est maintenant devenu positif car cet attribut ouvre sur le Nouveau-Monde repensé en fonction d'un approfondissement de l'altérité qui est en nous et en tous. Pour cela, il est utile de passer par une grande ville cosmopolite où les différences se croisent, dialoguent et créent du nouveau parfois à partir d'un local importé et recontextualisé:

[...] et où donc, si ce n'est à Paris, peut-on conquérir ce merveilleux territoire qu'on a hérité du Nouveau Monde et qui nous permet de circuler librement parmi les autres sans vouloir rivaliser avec eux, parce qu'un sauvage, faut-il le rappeler, ce n'est pas quelqu'un qui fuit les autres mais quelqu'un qui essaie de se rapprocher de cette part de lui-même qui lui échappe et qu'il retrouve parfois dans un arbre ou un visage (Yvon Rivard, Le siècle de Jeanne, p. 287).

Cette part de lui-même proche de l'affirmation de soi comme être singulier mentionnée aussi par James Baldwin ${ }^{6}$, un écrivain noir, gay et états-unien qui a longtemps vécu à Paris entre les deux guerres mondiales, ouvre sur la reconnaissance du nouveau en tant qu'étrangeté merveilleuse. Pour Rivard, la beauté repose sur la rencontre qui est reconnaissance de l'altérité car l'autre est en soi et ouvre à une dynamique qui évite la rivalité au sens de René Girard (« sans vouloir rivaliser avec eux », dit Yvon Rivard). Alors on évite le processus victimaire et le mécanisme de production de boucs émissaires, car l'autre est intérieur autant qu'extérieur. Dans l'attribution contemporaine de sauvage, la synonymie barbarie/extérieur/les autres n'est plus valide. Désormais, les Amériques, comme le montre Yvon Rivard, ne se perdent pas dans l'Europe et ne la rejettent pas non plus car elle est loin de représenter une forme de domination angoissante reléguant les Amériques au second plan. En effet, en séjournant à Paris, Alexandre, le personnage principal du Siècle de Jeanne, intègre le poids historique de l'Europe «car ne sont réels que les êtres, les lieux et les choses perdus et retrouvés» (p. 94). Point de dualisme donc, mais des réseaux de savoirs et de souvenirs, d'émotions et d'intelligence qui transforment les ancrages identitaires en 
des images de soi qui seyent aux habitants des Amériques s'inventant constamment en une capitalisation multicolore de cultures en processus de recontextualisation notamment grâce à l'accès pour tous au web. Par ces nouvelles technologies, ainsi, nous ne vivons pas dans un village global comme le supposait Marshall McLuhan mais dans une urbanité réseautée en un cerveau mondial.

\section{Le nouveau Nouveau Monde}

La rencontre au-delà des paradigmes dualistes transforme le social comme le souligne Yvon Rivard qui propose sa conception de l'éternité mobile de l'instant qui contient tout: « je m'entêtais à croire que les erreurs, les ratés et les horreurs de l'Amérique ne procèdent pas tant de la perte de la mémoire ou de l'effondrement de la culture européenne que de la résistance à cette nouvelle culture de l'instant, qui n'est pas dépourvue de mémoire, comme on le pense trop souvent, mais qui est au contraire la plus grande mémoire, la plus vivante, celle qui me rend à chaque instant contemporain du commencement et de la fin du monde» (p. 288). Cet instant, creuset de la beauté des rencontres par opposition à time is money comme aux canons historiques et à leurs stéréotypes excluants, rejoint la capacité de s'exposer au danger d'être en vie dans toute son acception éthique et humaine car « il faut maintenant tout recommencer, recommencer à voir, à sentir, à comprendre que ce monde et nous-mêmes n'avons pas d'âge puisque nous naissons ensemble à chaque instant et que chaque instant est une frontière mobile où se rencontrent tous les siècles passés et à venir» (p. 293). Comment mieux définir symboliquement les fascinations entre individus et groupes de cultures et d'origines différentes? Cette expérience est alors un des moteurs qui poussent les individus à échapper aux stéréotypes crus protecteurs de la communauté et à s'affirmer, à prendre le risque de devenir eux-mêmes, c'est-à-dire soi et l'autre dans le renouveau d'une affirmation de l'appartenance à soi qui est la liberté de toujours risquer d'être singulier avec les autres ${ }^{7}$. Chez Rivard, cette frontière mobile de l'instant couplée à une conscience de l'esthétique de la vie se rapproche de la frontier au sens d'espace non fini et ouvert mais dans une temporalité qui nait de la fusion de l'ici et du maintenant afin d'empêcher le monde de vieillir trop tôt. Mais la frontier de nos jours n'est plus l'espace présenté comme vide de l'Ouest ou du Grand Nord. C'est celui des créativités culturelles 
dans les rencontres urbaines productives entre des individus réseautés. Volonté de vivre dans le nouveau et le renouveau du Nouveau Monde, intensité, sensation de la démesure et de l'immensité retrouvée, attention à la vie, à la beauté dans l'exploration de la lenteur, voilà une attitude que ne renierait pas Tomson Highway parlant des Amériques dans Comparing Mythologies même s'il y ajouterait l'insertion historique, symbolique et spirituelle des Autochtones dans le territoire.

\section{Conclusion}

La rencontre a lieu entre les démunies dans La logeuse. Elles parviennent à dominer les passés qui les séparent pour collaborer dans un présent peu enthousiasmant. Pour cela, il a fallu qu'elles échappent à l'autoritarisme national ancré dans une histoire qui concerne surtout les gens nés sur le territoire et que la logeuse, Jeanne Joyal voulait imposer à toutes. Néanmoins, les clichés sont toujours là pour faire des dégâts. Ainsi, brusquement les immigrantes jettent à la tête de Rosa une série de clichés qui présentent les Québécois comme des nuls: «Ils conduisent comme des fous!...Ils n'apprennent rien à l'école! Ils baragouinent une langue qu'ils veulent imposer à tout le monde!...Ils ne comprennent rien, à part le hockey et les frites!» (p. 202). Et toutes de pulsionner en reprenant des préjugés fondés sur le ressentiment. Ce concert d'accusations « adressées à tous par tous» (p. 203) bouleverse Rosa car, de toute manière, elle ne comprenait rien au hockey et elle ne conduisait pas mais savait bien se conduire. Le ressentiment collectif surgit soudain dans le rejet de l'altérité. Autrement dit, comme dans le roman de Dany Laferrière, c'est le savoir institutionnalisé et figé commun au groupe qui mine les relations. Cela correspond bien à la théorie de René Girard qui souligne que le groupe se constitue sur l'exclusion d'un bouc émissaire pour contrôler la mimésis d'appropriation, c'est-à-dire le fait que tous luttent contre tous pour obtenir une place au soleil dans une société donnée.

Alors pour favoriser la rencontre, que faut-il faire? Il faut se déposséder des normes institutionnelles intégrées. Et parmi les normes, il y a les savoirs accumulés sur les autres, ce que l'on nomme des stéréotypes. Ceux-ci sont généralement projetés quand on veut, même de façon bien intentionnée, montrer que l'on sait déjà quelque chose sur l'autre. Celui-ci généralement ne se reconnaît pas dans ces phrases. En fait, il est nécessaire de 
manifester une discrète curiosité qui demande l'approche d'individu à individu. Celle-ci ne réussit pas forcément ou seulement jusqu'à un certain point comme on le voit chez Dany Laferrière. En effet, la Blanche et le Noir font l'amour ce qui est un progrès par rapport au rejet raciste, mais ils ne dorment pas ensemble. Il manque là une extension de la curiosité, ce qui veut dire, une volonté de sortir de sa zone de confort, d'explorer des parcours nouveaux dans une ville que tout un chacun transforme par ses habitudes en son village constitué de réseaux connus.

Ainsi, ce n'est que récemment que le roman au Québec explore les parcours des altérités en échappant à la collusion entre question sociale et question nationale pour ouvrir à des relations qui transgressent les clivages ethniques, linguistiques ou de classe. Dans ces romans la question sociale n'est plus uniquement politico-syndicale et contrôlée par les mâles. Elle est ouverte aux multiples aspirations de voix longtemps inaudibles. La question nationale, elle non plus, ne peut reposer sur un dualisme où un groupe fait bloc contre un autre ethniquement et linguistiquement. Elle est traversée de questions qui concernent ce que veut dire être citoyen dans une ville aux multiples visages et aux langues qui circulent, autrement dit dans des dynamiques interculturelles, celles dont parle Gérard Bouchard dans son ouvrage intitulé L'interculturalisme québécois.

\section{Referências}

ANGERS, François-Réal. Les révélations du crime ou Cambray et ses complices. Québec: sans éditeur, 1867.

ARAVIND ,Adiga. The White Tiger. New Delhi: HarperCollins India, 2008.

BOTCHORICHVILI, Elena. Seulement attendre et regarder. Montréal: Boréal, 2012.

BOUCHARD, Gérard. L'interculturalisme: un point de vue québécois. Montréal: Boréal, 2012.

CHAMBERLAND, Paul. Terre Québec suivi de L'afficheur hurle. Montréal: Typo, 2003. CHAUVEAU, Pierre-Joseph Olivier. Charles Guérin. Montréal: Fides, 1979.

CLARKE, George Elliott. Execution poems: the Black Acadian tragedy of "George and Rue". Wolfville (Nova Scotia): Gaspereau Press, 2001.

DE GASPÉ, fils, Philippe Aubert. L'influence d'un livre. Québec: Presses de l'Université Laval : 1964.

DE GASPÉ, père, Philippe Aubert. Les anciens Canadiens. Québec: Desbarats, 1867. 
DE GRANDPRÉ, Pierre. Dix ans de vie littéraire au Canada français. Montréal: Beauchemin, 1966.

DE NEVERS, Edmond. L'avenir du peuple canadien-français. Paris: H. Jouve, 1896.

DUPONT, Éric. La logeuse. Montréal: Marchand de feuilles, 2006.

FERRON, Jacques. Contes, Montréal: Hurtubise HMH, 1985.

GALE, Lorena. «Je me souviens», Voice of Her Own. Introduction de Sherill Grace, Toronto: Playwrights Canada Press, 2003.

HIGHWAY, Tomson. Comparing Mythologies. Ottawa: University of Ottawa Press, 2001. IMBERT, Patrick. 'Le père Goriot' au Canada: feuilleton et censure. In: L'année balzacienne, 1986, p. 237-246.

LACOMBE, Patrice. La terre paternelle. Montréal: Beauchemin et Valois, 1871.

LAFERRIÈRE, Dany. Comment faire l'amour avec un Nègre sans se fatiguer. Montréal: VLB, 1985.

MARION, Séraphin. Les lettres canadiennes d'autrefois. Ottawa: Éditions de l'Université, 1938.

MARTIN-MEUNIER, E. et Jean-Philippe WARREN. De la question sociale à la question nationale: la revue Cité Libre (1950-1963). In: Recherches sociographiques, vol. 39, n*23, 1998, p. 291-316.

MEMMI, Albert. Portrait du colonisé. Montréal: L'étincelle, 1972.

OUELLETTE-MICHALSKA, Madeleine. Du côté de l'Éden. In: L'Amour de la carte postale. Montréal: Québec/Amérique, 1987.

RIVARD, Yvon. Le siècle de Jeanne. Montréal: Boréal, 2005.

ROY, Gabrielle. Bonheur d'occasion. Montréal: Boréal, 2009.

RUSHDIE, Salman. The Firebird's Nest. The New Yorker, June 1997.

SARMIENTO, Domingo Faustino. Facundo. Paris: Stock, 1834 (1ère éd. 1845).

STRARAM, Patrick. le bison ravi. Montréal: l'hexagone/l'obscène nyctalope, 1972.

TRUDEL, Marcel. L'esclavage au Canada français. Montréal: Éditions de l'Horizon, 1963.

VALLIÈRES, Pierre. Nègres blancs d'Amérique. Montréal: Parti-pris, 1968. Wired, March 2000.

\section{Notes}

${ }^{1} \mathrm{PhD}$ (1974). Professeur éminent de l'Université d'Ottawa et Directeur de la chaire: Canada : enjeux sociaux et culturels dans une société du savoir.pimbert@uottawa.ca 
${ }^{2}$ Et contrairement à la gauche européenne, la gauche québécoise se tait sur les Autochtones. Comme le souligne Madeleine Ouellette-Michalska dans le chapitre intitulé «Du côté de l'Éden» de son recueil d'essai L'Amour de la carte postale (Montréal, Québec/Amérique, 1987, p. 149), cette gauche revendique pour les Québécois francophones « le statut de 'nègres blancs d'Amérique' » mais « il ne vient à personne l'idée de se dire Indien ». Ce ne sera que plus tard, notamment à travers les essais de la gauche contre-culture de Patrick Staram, un français émigré au Canada, que les Autochtones prendront figure plus idéale (Patrick Straram, le bison ravi, Montréal, l'hexagone/l'obscène nyctalope, 1972). Autrement dit, l'impensé généré par le dualisme touche tous les types de discours.

${ }^{3}$ Lorena Gale, Je me souviens, Voice of Her Own, Introduction de Sherill Grace, Toronto, Playwrights Canada Press, 2003, p. 65.

${ }^{4}$ Ibid., p. 166. L'Europe a perdu une bataille mais a gagné la guerre grâce aux Canadiens, aux États-Uniens et aux Anglais ainsi qu'à des petites armées en exil et des résistants à l'intérieur. Simultanément, elle a en effet perdu son pouvoir mondial face à la puissance des États-Unis et à la décolonisation. Donc, là aussi, le flou du mot perdre génère des angoisses intéressantes exprimées dans un discours littéraire elliptique qui permet de saisir les conditions des identités « incertaines » dont parle Ferron.

${ }^{5}$ Patrick Imbert, «'Le père Goriot' au Canada : feuilleton et censure », L'année balzacienne, 1986, p. 237-246.

${ }^{6}$ Un auteur des États-Unis qui constitue un intertexte important dans Comment faire l'amour avec un Nègre sans se fatiguer.

${ }^{7}$ Ce lien entre beauté, risque de devenir soi et liberté incluant la liberté d'entreprendre est aussi constatée dans de nombreuses œuvres contemporaines et dans des lieux où l'on parle de l'influence des Amériques. C'est ce qu'on constate chez l'écrivain de l'Inde Aravind Adiga. Dans son roman intitulé The White Tiger, il se consacre au passage d'une société traditionnelle à une société qui prend en partie son inspiration d'une mondialisation urbanisée pour laquelle les États-Unis et l'Amérique du Nord individualistes, riches et technologiques fascinent. Il évoque la vie d'un paysan pauvre du nord de l'Inde mais devenu entrepreneur à Bangalore, ce qui n'est pas sans rappeler un article du Magazine Wired faisant l'apologie de la réussite des membres des minorités dominés en Inde et trouvant leur inspiration dans le développement des États-Unis: «Kar wanted to do something else. After earning his MBA from the University of Bombay in 1983, he set out to knock down the caste system's barriers, using technology as his battering ram. 'I concur strongly with Thomas Jefferson' he says. 'I am more interested in the dreams of the future than the history of the past. I am the first in my family to do his own things'». Wired, March 2000, p. 158. Nous traduisons: Kar voulait faire autre chose. Après avoir obtenu son MBA de l'université de Bombay en 1983, il s'est donné pour mission de renverser les barrières du système de castes en utilisant la technologie comme son tambour de guerre. 'Je suis fortement d'accord avec Thomas Jefferson' dit-il. 'Je suis plus intéressé par les rêves du futur que par l'histoire du passé. Je suis le premier dans ma famille à faire ce que je veux'. Le personnage de Adiga affirme de ceux qui sont moins bien organisés que lui: «They remain slaves because they can't see what is beautiful in this world». Aravind Adiga, The White Tiger, New Delhi, HarperCollins India, 2008, p. 40. Nous traduisons: Ils demeurent des esclaves parce qu'ils ne sont pas capables de voir la beauté dans le monde» Voir aussi p. 276: "Iqbal, that great poet, was so right. The moment you recognize what is beautiful in this world, you stop being a slave". Nous traduisons: Le grand poète, Iqbal, avait raison. Le moment où l'on reconnaît la beauté dans le monde, on cesse d'être un esclave. Au sujet de la fascination par l'Amérique du Nord, voir aussi Salman Rushdie, "The Firebird's Nest » The New Yorker, June 1997 p. 35: «America... My country (India) is just like yours (The United States), he'd said, flirting. Big, turbulent, and full of gods. We speak our kind of bad English and you speak yours. And before you became Romans, when you were just colonials, our masters were the same. You defeated them before we did. So now you have more money than we do. Otherwise, we're the same".

Nous traduisons: Mon pays (l'Inde) est juste comme le vôtre (les États-Unis), dit-il, en flirtant. Grand, turbulent et plein de dieux. Nous parlons notre forme de mauvais anglais et vous parlez le vôtre. Et avant que vous ne deveniez des Romains, quand vous n'étiez que des colonisés, nos maîtres étaient les mêmes. Vous les avez défaits avant nous. Ainsi, vous avez maintenant plus d'argent que nous. Sinon nous sommes pareils. 